\title{
$\operatorname{CONF}-941144--108$
}

Note: This is a preprint of a paper submitted for publication. Contents of this paper should not be quoted or referred to without permission of the author(s).

Presented at the Fall Meeting of the Materials Research Society, Boston, Massachusetts, November 28-December 28, 1994 and published in

Structure and Properties of Interfaces in Ceramics, ed. by D. A. Bonnell, U. Chowdhry, and M. Rühle, Materials Research Society, Pittsburgh, PA

\section{MECHANISMS OF ENHANCED IONIC CONDUCTION AT INTERFACES IN CERAMICS}

D. Lubben and F. A. Modine

Solid State Division, Oak Ridge National Laboratory

P.O. Box 2008, Oak Ridge, Tennessee 37831-6030

\begin{abstract}
The submitted manuscript has been authored by a contractor of the U.S. Govemment under contract No. DE-AC05-840R21400. Accordingly, the U.S. Government retains nonexclusive, royalty-free license to publish or reproduce the published form of this coproduction, or allow ather to do 10 , for U.S. Contribution, or allow athers to do 20 , for U.S.
Government purposes."
\end{abstract}

\author{
SOLID STATE DIVISION \\ OAK RIDGE NATIONAL LABORATORY \\ Managed by \\ MARTIN MARIETTA ENERGY SYSTEMS, INC. \\ under \\ Contract No. DE-AC05-84OR21400 \\ with the \\ U.S. DEPARTMENT OF ENERGY \\ Oak Ridge, Tennessee \\ November 1994




\section{DISCLAIMER}

Portions of this document may be illegible in electronic image products. Images are produced from the best available original document. 


\title{
MECHANISMS OF ENHANCED IONIC CONDUCTION AT INTERFACES IN CERAMICS
}

\author{
D. LUBBEN and F. A. MODINE
}

Solid State Division, Oak Ridge National Laboratory, P.O. Box 2008, Oak Ridge, TN 378316030.

\section{ABSTRACT}

A large enhancement in the ionic conductivity of certain compounds occurs when the compound is produced as a composite material containing a finely-dispersed non-conductor such as $\mathrm{SiO}_{2}$ or $\mathrm{Al}_{2} \mathrm{O}_{3}$. This effect has been reported on for more than 20 years, and it is well established that the enhancement is associated with the presence of interfaces. The popular explanation has been based on a model which contends that the enhancement is due to a space-charge layer which forms to compensate a net charge layer at an interface. A different model proposes that extended defects such as dislocations and grain boundaries, either resulting from or stabilized by the interface, are responsible for the enhancement. This paper describes recent experiments which strongly support the latter model. The ionic conductivities of $\mathrm{LiI}$ and $\mathrm{CaF}_{2}$ thin films grown on sapphire(0001) substrates were monitored in-situ during deposition as a function of film thickness and deposition conditions. LiI films grown at $27^{\circ} \mathrm{C}$ exhibited a region of enhanced conduction - within $100 \mathrm{~nm}$ of the substrate and a lesser enhancement as the film thickness was increased further. This conduction enhancement was not stable but annealed out with a characteristic $\log$ (time) dependence. The observed annealing behavior was fit with a model based on dislocation motion which implies that the increase in conduction near the interface is due to extended defects generated during the growth process. LiI films grown at higher temperatures $\left(100^{\circ} \mathrm{C}\right)$ in order to reduce the grown-in defects showed no interfacial conduction enhancement. X-ray diffraction measurements suggest that these high-temperature LiI films nucleate as faceted epitaxial islands with a stable misfit dislocation density defined by the epitaxial relationship between the substrate and film. $\mathrm{CaF}_{2}$ films grown at $200^{\circ} \mathrm{C}$ showed a behavior similar to the $27^{\circ} \mathrm{C} \mathrm{LiI} \mathrm{films,} \mathrm{with} \mathrm{a}$ region of thermally unstable enhanced conduction that occurs within $10 \mathrm{~nm}$ of the substrate. Amorphous $\mathrm{Al}_{2} \mathrm{O}_{3}$ films deposited over the $\mathrm{CaF}_{2}$ layers created no additional enhancement but did increase the stability of the conduction, consistent with an extended defect model. Simultaneous deposition of $\mathrm{CaF}_{2}$ and $\mathrm{Al}_{2} \mathrm{O}_{3}$ produced films consisting of very-fine-grained $\mathrm{CaF}_{2}$ and particles of amorphous $\mathrm{Al}_{2} \mathrm{O}_{3}$ (5-10 nm grain and particle size) and a high defect density which was stable even well above the growth temperature. Measured conduction in the composite at $200^{\circ} \mathrm{C}$ was approximately 360 times that of bulk $\mathrm{CaF}_{2}$.

\section{INTRODUCTION}

The conductivity of many ionic conductors is increased by up to several orders of magnitude when the material is prepared as a composite with a finely-dispersed second phase. In most cases, the second phase consists of a non-conducting material such as $\mathrm{SiO}_{2}$ or $\mathrm{Al}_{2} \mathrm{O}_{3}$ which typically is considered to be insoluble in the host material under the fabrication conditions. Since the first report by Liang over twenty years ago $^{1}$, this effect has been observed in numerous systems. ${ }^{2-18}$ (See Reference 2 for a review through 1989.) Some of the largest enhancements have 
been observed in the alkali- and alkali-earth halides such as $\mathrm{CaF}_{2}^{9,12}$ and $\mathrm{Lil}^{1,4,8}$.

A considerable effort has been devoted to understanding this behavior. Most investigators assume that the enhancement occurs at the interface between the ionic conductor and (in the usual case) non-conductor and that classical impurity doping mechanisms cannot account for the very large enhancements which are often observed. On the other hand, the mechanism for the interfacial enhancement is not clear. The most popular explanation, introduced by Jow and Wagner ${ }^{3}$ and later developed extensively by Maier ${ }^{19-22}$ and others, is based on the space-charge theory of Kliewer and Kohler ${ }^{23-25}$. In this model, an interfacial enhancement is caused by an increase in the carrier concentration arising from a preferential occupation of interface sites by one species. The formation of a diffuse compensating layer of oppositely charged point defects provides the excess carriers responsible for the enhanced conduction. A different mechanism has been proposed by Dudney ${ }^{11}$, who calculated the maximum enhancement which could be expected from the space-charge effect for a variety of systems and showed that at least in some cases, in particular LiI and AgI, the observed enhancements are much larger than can be explained by the space-charge model. She also studied the conduction in $\mathrm{AgCl}$ and $\mathrm{AgCl}: \mathrm{Al}_{2} \mathrm{O}_{3}$ composites fabricated from molten $\mathrm{AgCl}$ and $\mathrm{Al}_{2} \mathrm{O}_{3}$ fiber papers ${ }^{13,14}$. Composites formed in this manner showed no enhancement as-prepared, but both the composites and the pure $\mathrm{AgCl}$ displayed enhanced conduction after deformation. The conductivity of the pure $\mathrm{AgCl}$ was restored to that of the undeformed material by annealing at $250^{\circ} \mathrm{C}$ while the composite retained some enhanced conduction (2-5 times that of the pure material) even after annealing to $400^{\circ} \mathrm{C}$. She concluded that deformation-induced defects which could be stabilized by the presence of the second phase were the source of the conduction enhancement.

In this paper we report the conductivity and structure of $\mathrm{LiI}$ and $\mathrm{CaF}_{2}$ films grown on sapphire substrates at different temperatures. Films grown at low temperatures $\left(\mathrm{LiI}\right.$ at $27^{\circ} \mathrm{C}, \mathrm{CaF}_{2}$ at $200^{\circ} \mathrm{C}$ ) had a conductivity profile which exhibited an enhancement near the interface. The conductivity was not stable but annealed out with a $\log (t i m e)$ dependence characteristic of dislocation climb. The deposition of amorphous $\mathrm{Al}_{2} \mathrm{O}_{3}$ over the $\mathrm{CaF}_{2}$ films produced no conduction enhancement but did decrease the rate at which the conductivity was reduced by annealing. On the other hand, composite films consisting of very-fine-grained $\mathrm{CaF}_{2}$ and $\mathrm{Al}_{2} \mathrm{O}_{3}$ particles (5-10 $\mathrm{nm}$ ) produced by simultaneous deposition of $\mathrm{CaF}_{2}$ and $\mathrm{Al}_{2} \mathrm{O}_{3}$ at $200^{\circ} \mathrm{C}$ had stable enhancements of 6300 in the near-interface region and 360 in the bulk film. LiI films grown at $100^{\circ} \mathrm{C}$ had a long incubation period during which essentially no conduction was observed. Once the conduction became measurable, it increased linearly with film thickness with no apparent interfacial enhancement and was completely stable at and above the growth temperature. X-ray diffraction measurements suggest that these LiI films nucleate as faceted epitaxial islands with a stable misfit dislocation density defined by the structural relationship between the substrate and film.

\section{EXPERIMENTAL PROCEDURE}

All experiments were performed in a UHV deposition system consisting of a stainless steel chamber equipped with a cryopump and a $450 \mathrm{l} / \mathrm{sec}$ turbomolecular pump. LiI (Aesar, $99.95 \%$ pure) and $\mathrm{CaF}_{2}$ was sublimated from $\mathrm{BN}$ and graphite crucibles, respectively. Growth rates varied from 0.25 to $2.5 \mathrm{~nm}$ per minute, and all experiments were carried out at a pressure $<10^{-9}$ Torr. The film thickness was continuously monitored with a quartz-crystal ratemonitor. The substrate was fixed to a $\mathrm{Nb}$ block which could be radiatively heated to temperatures in excess of $500^{\circ} \mathrm{C}$. The substrate temperature was monitored by a Chromel-Alumel thermocouple embedded in the 
block, which was used as feedback for a control circuit to maintain a constant temperature during deposition and to achieve a constant temperature ramp during temperature-dependent conductivity measurements.

The substrates used were sapphire(0001) wafers polished on one side obtained from Kappler Crystal Optics, Inc. All substrates were ultrasonically cleaned in acetone, methanol and deionized water and subsequently annealed in air for 48 hours to remove polishing damage. After slow furnace cooling, interdigital $\mathrm{Cr}$ :Au electrodes were deposited onto the substrates using standard photolithographic techniques. The electrode geometries, which were chosen based on the expected conductivities, resulted in geometric factors $\mathrm{l} / \mathrm{w}$ of 2530 for $\mathrm{CaF}_{2}$ and 167 for $\mathrm{LiI}$. The electrodes were connected via Ta clips to $\mathrm{Cu}$ wires attached to a Ni-wire feedthrough on the vacuum jacket.

A Hewlett-Packard model 4192A LCR meter was used for the impedance measurements. A $0.1 \mathrm{~V}$ signal was applied across the electrodes and the resulting current was amplified by a current-mode preamplifier (PAR model 184) set to a gain of $10^{5}-10^{7}$ which was attached to the vacuum feedthrough. The signal from the preamplifier was input to the LCR meter which measured the gain and phase of the current with respect to the input signal. These values were used to compute the real and imaginary parts of the impedance. During deposition, gain and phase were determined for up to 7 frequencies at half-decade intervals between $10 \mathrm{~Hz}$ and $10 \mathrm{kHz}$ every 20 seconds. Conductance values obtained over the frequency range $32 \mathrm{~Hz}<f<3.2 \mathrm{kHz}$ were nearly identical. Analysis of the impedance spectra revealed that the conductance measured at lower frequencies contained a contribution from the electrodes while frequencies above $3.2 \mathrm{kHz}$ resulted in an appreciable contribution from the long leads required to reach the substrate from the vacuum jacket.

XRD measurements were carried out on a Scintag diffractometer utilizing $\mathrm{Cu} \mathrm{K} \alpha$ radiation. All spectra were obtained after aligning the sample using the sapphire (0006) reflection. For the measurements on LiI, a series of films without electrodes were grown under identical conditions of growth rate and temperature. After deposition, the LiI samples were coated with Si-based vacuum grease which was pressed onto the surface together with a Kapton sheet in the load-lock chamber before exposing to the atmosphere in order to reduce water contamination (uncoated films would completely disintegrate in a few seconds). Rocking curves of the major peaks were also obtained, but owing to the presence of both $\mathrm{K} \alpha_{1}$ and $\mathrm{K} \alpha_{2}$ lines (the source utilized a Ni filter but was not monochromatized) high resolution rocking curve measurements were not possible.

\section{EXPERIMENTAL RESULTS}

\section{LiI}

Figure 1 shows the conductance of a LiI film grown on a sapphire(0001) substrate as a function of thickness. After an initial incubation period during which it rises only slowly, the conductance of the film increases rapidly before leveling off at a thickness of approximately 100 $\mathrm{nm}$. The conductivity of the film, obtained from the slope of the curve after correcting for the geometric factor of the electrodes, reaches a maximum of $\sim 5.5 \times 10^{-6} \mathrm{~S} / \mathrm{cm}$ near the interface and $\sim 1.5 \times 10^{-7} \mathrm{~S} / \mathrm{cm}$ in the bulk of the film.

The conductivity of the films was not stable at the growth temperature and in fact annealed appreciably even during growth. After deposition was stopped, the conductance was continuously monitored for 5 days ( 7200 minutes) to examine this behavior. The conductivity is plotted as a function of time in Figure 2, where both the film thickness $(250 \mathrm{~nm})$ and geometric factors have 


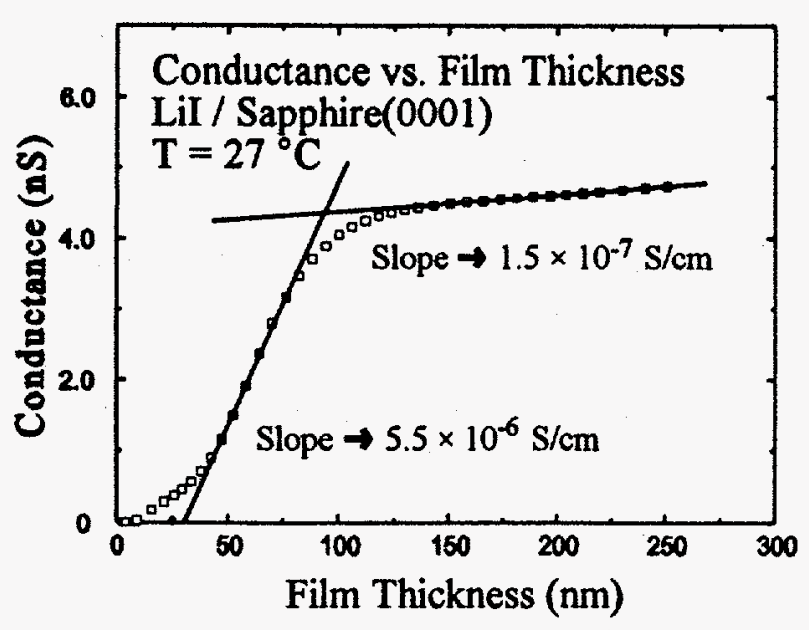

Figure 1. Conductance measured for a $\mathrm{LiI}$ film grown at a rate of $0.25 \mathrm{~nm}$ per minute on a sapphire $(0001)$ substrate at $27^{\circ} \mathrm{C}$.

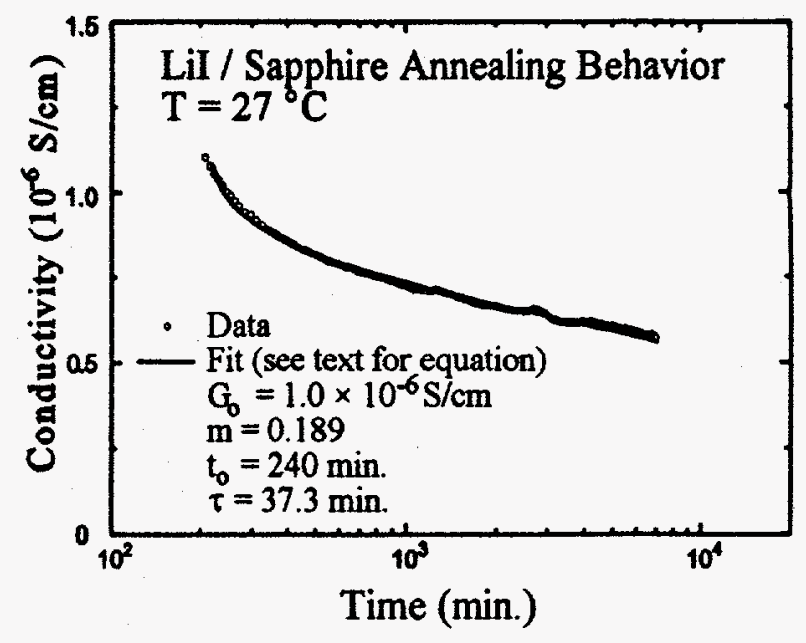

Figure 2. Conductivity of a 0.250 -nm-thick LiI film grown on sapphire $(0001)$ at $27^{\circ} \mathrm{C}$ as a function of time after growth.

been taken into account. The data points correspond to the measured conductivity which is observed to decrease with a $\log ($ time $)$ dependence. The solid line is a fit to the data using the model described in the discussion.

The temperature dependence of the conductivity is plotted in Figure 3. This film was grown under conditions identical to those in Figures 1 and 2 but was not annealed for an extended period. Instead, the temperature was ramped from room temperature to $100^{\circ} \mathrm{C}$ several times. The conduction reduction due to annealing accelerated as the temperature was raised, but on cooling a straight-line behavior was observed. The slope corresponds to an activation energy of $0.47 \mathrm{eV}$.

Films grown at elevated temperature are markedly different. Figure 4 is a plot of the measured conductance as a function of thickness for a LiI film grown on sapphire $(0001)$ at $100^{\circ} \mathrm{C}$. In this case, no conduction is observed initially until it turns on abruptly at $50 \mathrm{~nm}$. From this point, the conduction increases linearly with thickness for the rest of the deposition. The conductivity derived from the slope of the film and accounting for the electrode geometry is $\mathbf{3 . 2}$ $\times 10^{-5} \mathrm{~S} / \mathrm{cm}$.

Not only is the deposition profile of the conductivity different from those of films grown at lower temperature, but the conductivity of this film remains constant for extended periods at the growth temperature. The conductivity of the film remained unchanged over a period of 5 days at the growth temperature. Moreover, the conductivity was unchanged after ramping the temperature from 25 to $120^{\circ} \mathrm{C}$ and back. The activation energy extracted from the Arrhenius plot was $\sim 0.75 \mathrm{eV}$, considerably higher than that of the room temperature film.

The XRD spectrum ( $2 \theta$ scan) of room-temperature $\mathrm{LiI}$ revealed a strong $(002)$ orientation with only peaks from the (111) and (002) [along with the (222) and (004)] reflections. For hightemperature films, only (111) and (222) reflections were observed. The rocking curve of the (002) peak from the $27^{\circ} \mathrm{C}$ film had a rather broad profile with a full width at half maximum (FWMH) 


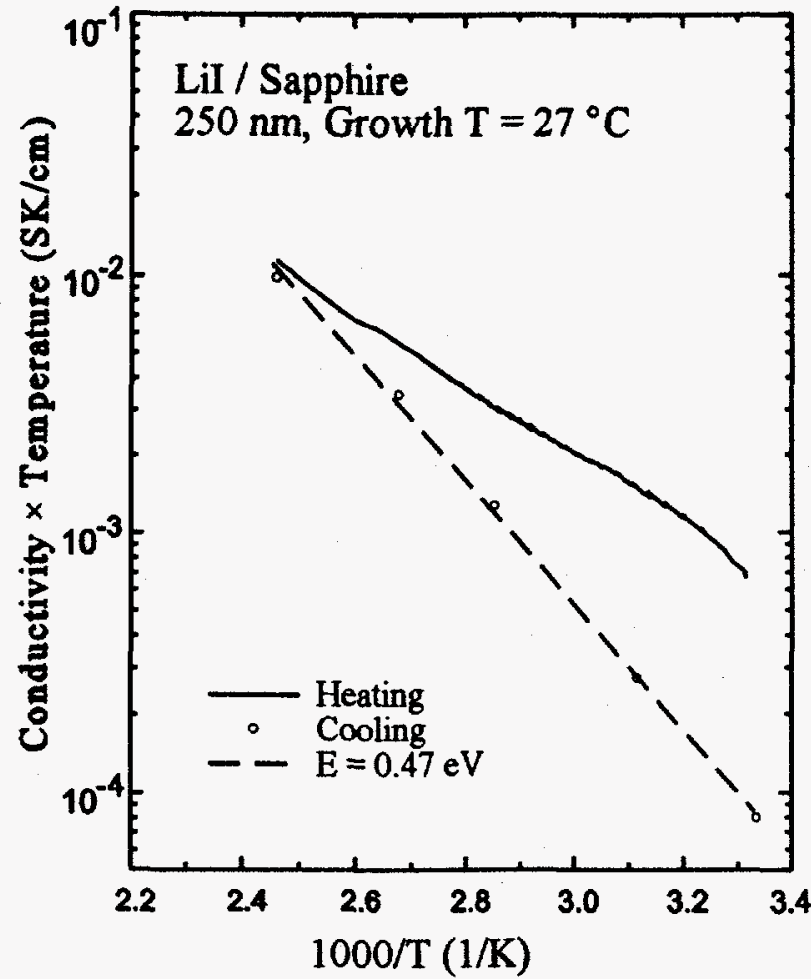

Figure 3. Temperature dependence of the conductivity of a 250 -nm-thick LiI film grown on sapphire $(0001)$ at $27^{\circ} \mathrm{C}$.

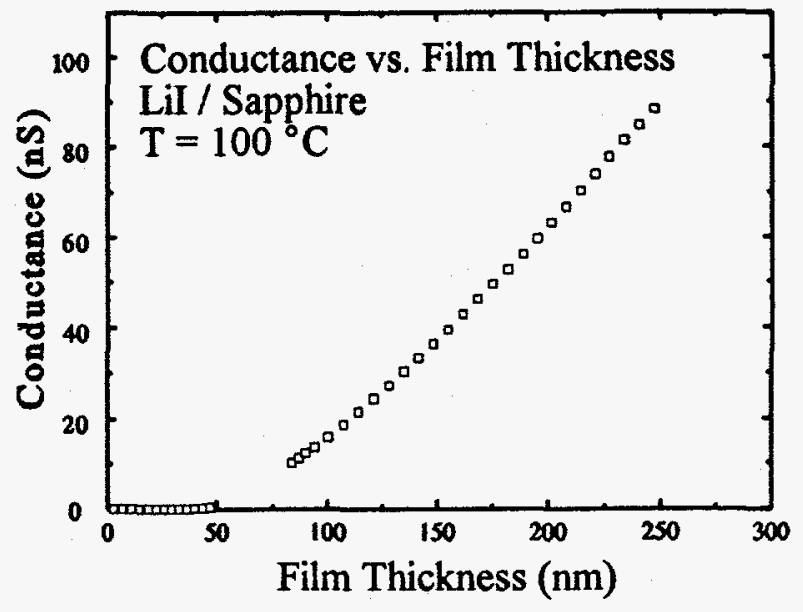

Figure 4. Conductance measured for a LiI film grown at a rate of $0.25 \mathrm{~nm}$ per minute on a sapphire $(0001)$ substrate at $100^{\circ} \mathrm{C}$.

of about 1.8 degrees and a large tail. The rocking curve of the (111) peak for the $100^{\circ} \mathrm{C}$ film had a much sharper profile with a FWHM of 0.38 degrees and no tail.

\section{2. $\mathrm{CaF}_{2}$}

Figure 5 shows the conductance versus the thickness of a $\mathrm{CaF}_{2}$ film on $\mathrm{Al}_{2} \mathrm{O}_{3}(0001)$. Beyond the initial incubation region, an enhanced conductance extends $\sim 10 \mathrm{~nm}$ from the interface after which the conductance increases with thickness at a reduced rate. As for the LiI films, the conductance is not stable but anneals out with a $\log ($ time $)$ dependence. Arrhenius plots of the conductance for $\mathrm{CaF}_{2}$ films that had annealed sufficiently to give reproducible results gave activation energies in the range $0.6 \pm 0.1 \mathrm{eV}$ and disclosed a definite tendency for the activation energy to increase due to annealing. For some films, Au electrodes were deposited on top in order to measure the conductivity perpendicular to the interface. These measurements gave a value of conductivity of $6 \times 10^{-10} \mathrm{~S}^{-\mathrm{cm}^{-1}}$ and an activation energy of $1.05 \mathrm{eV}$, very close to the reported values of $3.4 \times 10^{-10} \mathrm{~S}-\mathrm{cm}^{-1}$ and $1 \mathrm{eV}$. [Refs 9, 26, 27] 


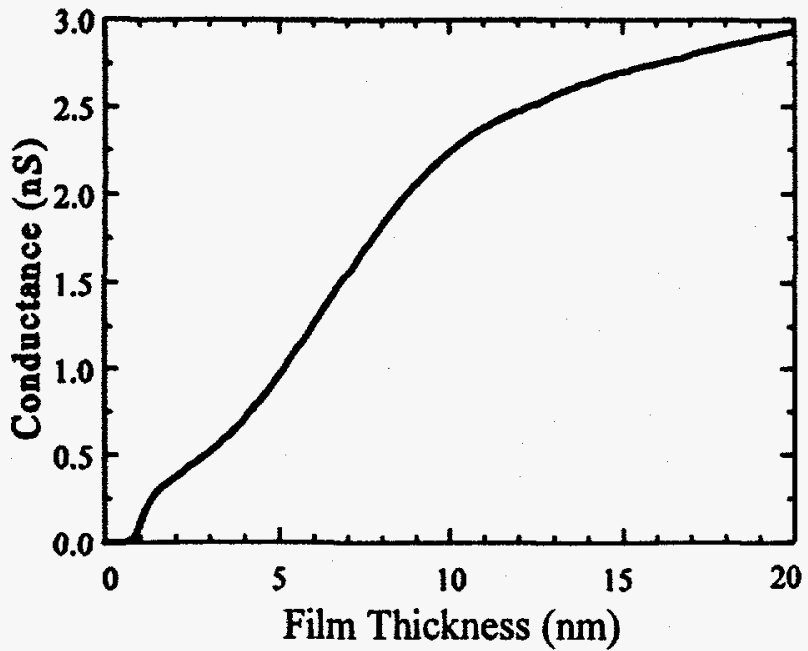

Figure 5. Conductance of a $\mathrm{CaF}_{2}$ film grown at a rate of $0.25 \mathrm{~nm}$ per minute on a sapphire $(0001)$ substrate at $200^{\circ} \mathrm{C}$.

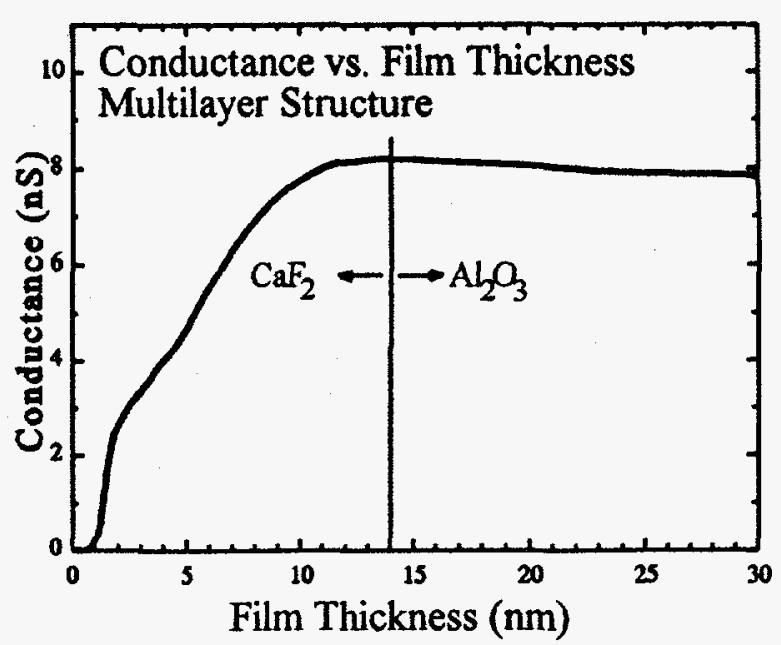

Figure 6. Conductance of a $\mathrm{CaF}_{2}$ film grown to a thickness of $14 \mathrm{~nm}$ on sapphire $(0001)$ and capped with a film of $\mathrm{Al}_{2} \mathrm{O}_{3}$. The $\mathrm{CaF}_{2}$ and $\mathrm{Al}_{2} \mathrm{O}_{3}$ were grown at rates of 1.2 and $0.8 \mathrm{~nm}$ per minute, respectively.

\section{Multilayer and composite films}

Figure 6 shows measurements made on a $\mathrm{CaF}_{2}$ film grown on an $\mathrm{Al}_{2} \mathrm{O}_{3}(0001)$ substrate and capped with a film of amorphous $\mathrm{Al}_{2} \mathrm{O}_{3}$. There is no conduction enhancement at the $\mathrm{CaF}_{2}-$ $\mathrm{Al}_{2} \mathrm{O}_{3}$ interface when the alumina is grown on the $\mathrm{CaF}_{2}$. Moreover, the addition of alumina thermally stabilizes the conductance of the underlying $\mathrm{CaF}_{2}$ film. The conductance of the $\mathrm{CaF}_{2}$ film is higher than that shown in Fig. 5 because the deposition rate was higher (see discussion).

In Fig. 7, the electrical conductance of a film of pure $\mathrm{CaF}_{2}$ is compared to that of a composite film containing about 10 mole percent $\mathrm{Al}_{2} \mathrm{O}_{3}$. The thickness dependence of the conductance of the pure $\mathrm{CaF}_{2}$ film differs from that shown in preceding figures because of the higher deposition rate. At low deposition rates, the conductance increases monotonically with film thickness. At a high growth rate, the conductance increases to a maximum and then decreases as the interface conduction is reduced by thermal annealing. A peak in the conductance is seen when a film is deposited at a rate which is fast compared to the rate of decrease due to thermal annealing. The conductance of the pure $\mathrm{CaF}_{2}$ film remains nearly constant as the thickness increases from about 50 to $800 \mathrm{~nm}$ because the conductance adjacent to the interface dwarfs the conductance away from the interface. The composite film is much more conductive than pure $\mathrm{CaF}_{2}$ far from the interface. A comparison of the slope of the conductance curve with the bulk conductivity of pure $\mathrm{CaF}_{2}$ discloses that the addition of $\mathrm{Al}_{2} \mathrm{O}_{3}$ enhances the conductivity by about 2.5 orders of magnitude. The $\mathrm{Al}_{2} \mathrm{O}_{3}$ also increases the thermal stability. 


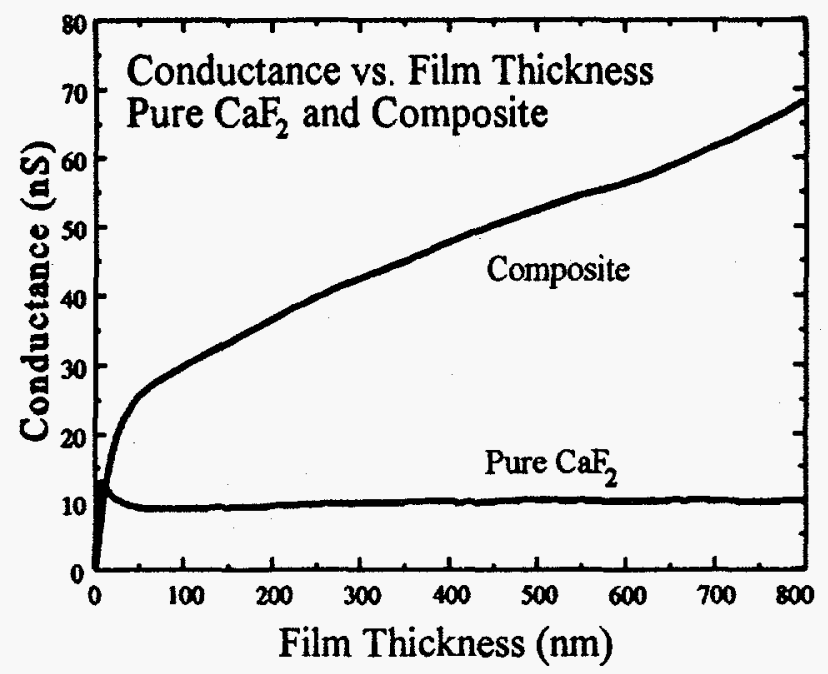

Figure 7. Conductance of a composite film of $\mathrm{CaF}_{2}$ with 10 mole percent $\mathrm{Al}_{2} \mathrm{O}_{3}$ compared to a film of pure $\mathrm{CaF}_{2}$. The composite and pure films were grown at $200^{\circ} \mathrm{C}$ at 4.3 and $5.2 \mathrm{~nm}$ per minute, respectively.

\section{DISCUSSION}

\section{Low-temperature films}

Measurements on single-crystal Lil have shown that conduction occurs by motion of $\mathrm{Li}$ vacancies whose formation and migration enthalpies are 1.06 and $0.43 \mathrm{eV}$, respectively. ${ }^{27}$ Similar results have been obtained for polycrystalline material in the extrinsic region ${ }^{28,29}$. Room temperature conductivities for nominally pure material $(10-100 \mathrm{ppm}$ of divalent impurities such as $\mathrm{Mg}, \mathrm{Fe}$ and $\mathrm{Ca}$ ) have been reported to be between $2 \times 10^{-8}$ and $3 \times 10^{-7} \mathrm{~S}-\mathrm{cm}^{-1}$. [Ref. 29-31] Polycrystalline $\mathrm{CaF}_{2}$ exhibits an activation energy of $0.9-1.0 \mathrm{eV}$ in the extrinsic region and about $2.0 \mathrm{eV}$ in the intrinsic region, while heterogeneously doped material has an activation energy of about $0.7 \mathrm{eV},{ }^{9,26-28}$

The films we grew at low temperatures $\left(\mathrm{LiI}\right.$ at $27^{\circ} \mathrm{C}, \mathrm{CaF}_{2}$ at $200^{\circ} \mathrm{C}$ ) showed very different conduction behavior in the regions adjacent to and far from the interface. In LiI, almost no conduction is observed in the first $10 \mathrm{~nm}$ of deposition which is probably due to the formation of islands during the initial stages of film growth. The onset of measurable conduction at $\sim 10$ $\mathrm{nm}$ is evidence for the beginning of island coalescence. Between 20 and $40 \mathrm{~nm}$ the measured conduction increases and, above $40 \mathrm{~nm}$, increases much more rapidly. The measured conductivity obtained from the slope of the curve in the steepest region is $5.5 \times 10^{-6} \mathrm{~S} / \mathrm{cm} 1$, which is considerably higher than bulk LiI. In the region away from the interface, the conductivity increases nearly linearly with a slope of $\sim 1.5 \times 10^{-7} \mathrm{~S} / \mathrm{cm}$, well within the range of reported values for bulk LiI. The conductivity in the near interface region with the largest slope is a factor 
of -40 times that in the bulk film. The $\mathrm{CaF}_{2}$ films have a conductance profile which is qualitatively similar to Lil. After the initial incubation, the enhanced region extends approximately $10 \mathrm{~nm}$ from the interface. The conductance derived from the slopes in the near-interface and bulk regions in Figure 6 is $1.2 \times 10^{6}$ and $2.0 \times 10^{-7} \mathrm{~S}^{-\mathrm{cm}^{-1}}$, respectively. Both are considerably higher than the bulk value $\left(3-6 \times 10^{-10} \mathrm{~S}-\mathrm{cm}^{-1}\right)$.

It should be noted here that the conductivity values determined for the interface and bulk during growth are complicated by the simultaneous annealing. The increase we measure is actually a convolution of the increase due to the addition of conducting material and the decrease due to annealing. The measured value will therefore depend on the actual as-deposited conductivity as well as the deposition and annealing rates. The annealing effectively reduces the measured value of bulk conductivity, although the effect is difficult to quantify. This effect is the reason for the differences in the measured conductances for the $\mathrm{CaF}_{2}$ films in Figures 5, 6 and 7 .

Maier ${ }^{21}$ has calculated the vacancy conductance for an interfacial space-charge layer:

$$
G=2 e \lambda\left(\frac{w}{l}\right) U_{v} \sqrt{N_{0} N_{\infty}}=\left(2 \epsilon \epsilon_{0} k T N_{0}\right)^{1 / 2} U_{v}\left(\frac{w}{l}\right)
$$

where

$$
\lambda=\left(\epsilon \epsilon_{0} k T / 2 e^{2} N_{\infty}\right)^{1 / 2}
$$

is the Debye length, $U_{V}$ is the vacancy mobility, $N_{0}$ and $N_{\infty}$ are the vacancy concentrations at the interface and in the distant bulk, and the factor $(w / l)$ is contributed by the electrode geometry.

We can compare our results to predictions from this space charge model by assuming a space-charge layer is responsible for the interfacial enhancement. For LiI, $\mathbf{N}_{\infty}$ may be calculated from the measured mobility of $4 \times 10^{-7} \mathrm{~cm}^{2} / \mathrm{V} / \mathrm{s}$ (Ref. 30) and the bulk conductivity we obtain from the slope in the region far from the interface $\left(1.5 \times 10^{-7} \mathrm{~S} / \mathrm{cm}\right)$. Using this value for $\mathrm{N}_{\infty}$ $\left(2.3 \times 10^{18} \mathrm{~cm}^{-3}\right)$ and inserting $\epsilon=8.2$ yields $\lambda=1.6 \mathrm{~nm}$, which implies an enhanced-conduction layer thickness of $3.2 \mathrm{~nm}$. This is a factor of about 30 too small for our observations. For $\mathrm{CaF}_{2}$. the bulk conductivity at $200^{\circ} \mathrm{C}\left[\sigma=6 \times 10^{-10} \mathrm{~S} / \mathrm{cm}\right]$ and the mobility of the fluorine ion vacancy ${ }^{32}\left[\mathrm{U}_{\mathrm{v}}=(220 / \mathrm{T}) \exp (-5920 / \mathrm{T})\right]$ imply $\mathrm{N}_{\infty}=2 \times 10^{15} / \mathrm{cm}^{3}$. This $\mathrm{N}_{\infty}$ together with the dielectric constant ${ }^{33}[\epsilon=7]$ yield an effective thickness of $2 \lambda=114 \mathrm{~nm}$, which is an order of magnitude too large.

It could be argued that the incorrect estimation of the enhanced layer thickness is due to the effects of annealing on the conductivity measurement. However, it seems unlikely that this could explain such a large discrepancy. We note that while magnitude of the enhancement we measure in LiI is larger than that reported by Schreck ${ }^{34}$, which could be qualitatively explained by a difference in annealing, there is virtually no discrepancy in the measured thickness of the enhanced conduction region.

An even bigger problem with the application of the space-charge theory to our results is the instability of the conduction enhancement. Nothing in the theory can account for the decay over long times of the conduction. On the other hand, a model developed by Johnston ${ }^{35}$, which associates the conduction increase of plastically deformed material with an increase in the number of piled-up dislocations, fits our results quite well. In thin films, dislocations can be generated during deposition by either lattice or thermal mismatch with the substrate or by growth conditions. Annealing reduces the stress by allowing dislocations to climb from the pileups which results in a reduced conduction. 
Dislocation theory describes the rate of escape of $n$ piled-up dislocations by the equation

$$
\frac{d n}{d t}=-v \exp _{1}-W / k T \text {, }
$$

where $\nu$ is an attempt frequency and $W$ is an activation energy for dislocation climb. Mott $^{(36,37)}$ suggests an activation energy of the form

$W=W_{0}-\gamma S b^{3} n$

where $W_{0}$ is the activation energy for climb for a free dislocation, $b$ is the dislocations' Burgers vector, $S$ is the yield stress and $\gamma$ is a constant of order 1. Solving Equations (3) and (4) and assuming that the conduction is linearly related to the number of dislocations in a pileup gives

$G=G_{o}\left(n / n_{\partial}\right)=G_{o}\left\{1-m \log \left[1+\left(t-t_{\partial}\right) / \tau\right]\right\}$

where

$m=k T / n_{o} \gamma S b^{3}$

and

$\tau=\left(k T / \nu \gamma S b^{3}\right) \exp \left(W_{d} / k T-1 / m\right)$

Here $n_{0}$ is the initial number of dislocations in a pileup, $t$ is the time and $T$ the absolute temperature. Although the yield stress for Lil has not been reported, we can estimate it from equation (6) and the fitted value of $m$ (see Figure 2) if we assume that $n_{0}$ is given by the interface-to-bulk enhancement ratio $(40)$. Taking $\gamma=1$ and $b=4.24 \AA$ (the $\mathrm{Li}-\mathrm{Li}$ spacing in the cubic structure) gives $S=7.2 \times 10^{7}$ dynes-cm ${ }^{-2}$. Using this value for $S$ and assuming $v=3 \times 10^{13}$ (Ref 38), we can use equation (7) to show that $W_{0}=1.2 \mathrm{eV}$. Fits of Eq. (5) were made for $\mathrm{CaF}_{2}$ assuming a yield stress ${ }^{23}$ of $1.5 \times 10^{8}$ dynes $/ \mathrm{cm}^{2}$ (Ref 39) and a Debye frequency of $10^{13} \mathrm{~Hz}$. This resulted in an activation energy for dislocation climb of $2.0 \pm 0.1 \mathrm{eV}$ and a value of $6 \times 10^{2}$ for the initial number of dislocations in a pileup.

The fitted values are reasonable. The yield stress of $\mathrm{Lil}$ is unknown, but we obtain a value comparable to that reported for $\mathrm{CaF}_{2}$. Since dislocation climb occurs by vacancy motion we might expect $W_{0}$ to be equal to the vacancy migration energy obtained from conduction measurements. However, dislocation climb will require a combination of both $\mathrm{Li}$ and I vacancy migration so we expect $W_{0}$ to be larger. The values 1.2 and $2.0 \mathrm{eV}$ seem reasonable.

\section{Multilayer and composite films}

Growth of $\mathrm{Al}_{2} \mathrm{O}_{3}$ on top of $\mathrm{CaF}_{2}$ film produces a composite structure with two planar interfaces. If a space charge layer stems from a reaction, there should be some symmetry at the $\mathrm{CaF}_{2}-\mathrm{Al}_{2} \mathrm{O}_{3}$ interfaces. However, the second interface induced no increase in the conductance of the film but did increase the thermal stability. Both results are consistent with a dislocationinduced enhancement mechanism since although no additional dislocations will be produced in 
the $\mathrm{CaF}_{2}$ by the presence of the cap, motion of those already present will be impeded by removal of the free $\mathrm{CaF}_{2}$ surface.

The composite $\mathrm{CaF}_{2}: \mathrm{Al}_{2} \mathrm{O}_{3}$ film of Fig. 7 was grown under similar conditions to the $\mathrm{Al}_{2} \mathrm{O}_{3}$ capped film of figure 6 except that the deposition of the two materials was performed simultaneously instead of sequentially. The only other difference was that the growth rate of the $\mathrm{CaF}_{2}$ was higher in the composite. Moreover, $\mathrm{x}$-ray photoelectron spectroscopy analysis revealed no difference in the chemical nature of the films. Thus it is reasonable to assume that they are chemically the same - the only difference is the way in which the $\mathrm{CaF}_{2}$ and $\mathrm{Al}_{2} \mathrm{O}_{3}$ is distributed. However, the microstructure is completely different. XRD and electron microscopy revealed that the pure $\mathrm{CaF}_{2}$ was polycrystalline with a (111) preferred orientation and grain sizes on the order of several thousand angstroms. The composite film consisted of very-fine randomly-oriented $\mathrm{CaF}_{2}$ grains and $\mathrm{Al}_{2} \mathrm{O}_{3}$ particles (5-10 nm grain and particle size) which resulted in very broad XRD peaks with a FWHM $>1$ degree. The conductivity of the film represents an enhancement over the bulk $\mathrm{CaF}_{2}$ value by a factor of 360 and an improvement over previously reported ${ }^{9,26,27}$ dispersed phase materials by a factor of 7 .

From the results for the multilayer film we would expect no enhancement in this composite based on the space-charge theory, since no enhanced conduction was present at the second interface. However, once again a microstructural interpretation can be given since. A large dislocation density must exist in a film with such a microstructure, and the very fine $\mathrm{Al}_{2} \mathrm{O}_{3}$ particles will act to pin both grain boundaries and dislocations and hence lead to a stable conduction enhancement.

\section{High-temperature films}

The data in Figure 4 show that in Lil films grown at elevated temperature the interfacial enhancement is completely absent and the conduction, once it commences, increases linearly with thickness with a corresponding conductivity of $3.2 \times 10^{-5} \mathrm{~S} / \mathrm{cm}$. This conductivity was completely stable at the growth temperature. In fact, after ramping the temperature to well above the growth temperature $\left(125^{\circ} \mathrm{C}\right)$ there was still no observed decrease. Moreover, the activation energy for conduction of $0.75 \mathrm{eV}$, much higher than for the low-temperature film, was obtained even at room temperature.

The XRD shows that the microstructure is quite different for films grown at different temperatures which is certainly not unusual. The change in orientation can be attributed to competing effects. On the one hand, films tend to grow with planar faces that possess low surface energies. In ionic crystals, the overwhelming consideration is whether a given plane has a net dipole moment so that the Madelung sums diverge. ${ }^{40}$ Such polar surfaces are quite unstable. On the other hand, if a close epitaxial relationship exists between the substrate and film there will be a strong driving force for films to orient themselves in a way that provides the best lattice matching. For films in which a polar surface has the best lattice match to the substrate, epitaxial films typically grow as faceted islands whose surfaces consist of non-polar planes. In $\mathrm{CaF}_{2}$, which can be epitaxially grown on $\mathrm{Si}$, smooth growth is common on the (111) surface, which is nonpolar, while growth on the polar (011) and (001) surfaces is often faceted..$^{40}$

Our Lil films all possess a cubic structure. No evidence for a hexagonal phase, which has been reported earlier for Lil films deposited on sapphire ${ }^{37,38}$, was observed. In cubic LiI the (111) surface is polar while the (001) is not. At low temperatures, where surface mobility is low, formation of epitaxial structures is more difficult, and the films possess the (002) (low energy surface) orientation. At elevated temperatures, higher surface mobilities allow the film to orient 
itself with respect to the substrate. This results in a lower interfacial energy but a large surface energy which drives the film to form facets. Evidence for faceting is seen in the very long incubation times before the onset of conduction as well as the somewhat cloudy appearance of the LiI films grown at $100^{\circ} \mathrm{C}$ compared to the colorless appearance of the low-temperature films.

It is not surprising that LiI(111) would grow preferentially on sapphire(0001). To begin with, both surfaces have a hexagonal symmetry. While there is at first glance a large apparent mismatch between the two surfaces, a close match can be made by aligning the LiI(01-1) direction with the sapphire(2-1-10). Then the spacing between second-nearest-neighbors along the LiI (011) on the $\mathrm{Li}$ (or I) sublattice (8.485 $\AA$ ) matches closely with the spacing between third-nearestneighbors along the sapphire $(2110)$ on the $\mathrm{O}$ sublattice $(8.241 \AA)$. The lattice mismatch associated with this configuration is $2.6 \%$.

We believe our films nucleate as faceted epitaxial islands and that no conduction is measured until the islands coalesce. Once a continuous film is present, both the conductance and the continuous-film thickness increase linearly. The film has a stable dislocation density that is defined by the lattice mismatch between the substrate and film.

\section{CONCLUSIONS}

In all the systems we have studied we have seen no evidence for enhanced ionic conduction resulting from a space-charge layer at the film / substrate interface. We have also shown that strong and stable enhancements can be produced that can be attributed to microstructure. While we cannot say that space-charge related conduction enhancement is not present in any composite system, it is clear that microstructural effects are of major importance in some materials and should not be ignored when trying to model ionic conduction in composites.

\section{ACKNOWLEDGEMENTS}

This research was supported by the Division of Materials Sciences, U.S. Department of Energy, under contract DE-AC05-84OR21400 with Martin Marietta Energy Systems, Inc.

\section{REFERENCES}

1. C. C. Liang, J. Electrochem. Soc. 120, 1289 (1973).

2. N. J. Dudney, Ann. Rev. Mater. Sci. 19, 103 (1989).

3. T. Jow and J. B. Wagner, Jr., J. Electrocem. Soc. 126, 1963 (1979).

4. J. B. Phipps, D. L. Johnson, and D. H. Whitmore, Sol. State Ionics 5, 393 (1981).

5. K. Shahi and J. B. Wagner, Jr., J. Electrochem. Soc. 128, 6 (1981).

6. K. Shahi and J. B. Wagner, Jr., Sol. State Ionics 7, 119 (1982).

7. O. Nakamura and J. B. Goodenough, Sol. State Ionics 7, 119 (1982).

8. F. W. Poulsen, N. H. Andersen, B. Kindl, and J. Schoonman, Sol. State Ionics 9\&10, 119 (1983).

9. T. L. Wen, R. A. Huggins, A. Rabenau, and W. Weppner, Rev. Chim. Min. 20, 643 (1983).

10. M. R.-W. Chang, K. Shahi, and J. B. Wagner, Jr., J. Electrochem. Soc. 131, 1213 (1984).

11. N. J. Dudney, J. Am. Ceram. Soc. 68, 538 (1985). 
12. S. Fujitsu, M. Miyayama, K. Koumoto, H. Yanagida, and T. Kanazawa, J. Mater. Sci. 20, 2103 (1985).

13. N. J. Dudney, J. Am. Cer. Soc. 70, 65 (1987).

14. N. J. Dudney, Sol. State Ionics 28-30, 1065 (1988).

15. U. Lauer and J. Maier, Sol. State Ionics 51, 209 (1992).

16. Y. Saito, K. Ato, T. Asai, H. Kageyama, and O. Nakamura, Sol. State Ionics 53-56, 728 (1992).

17. S. Narender Reddy, A. Sadananda Chary, and T. Chiranjivi, Sol. State Ionics 66, 131 (1993).

18. F. A. Modine and D. Lubben, J. Appl. Phys. 74, 2658 (1993).

19. J. Maier, Phys. Stat. Sol. B123, K89 (1984).

20. J. Maier, Sol. State Ionics 23, 59 (1987).

21. J. Maier, Phys. Stat. Sol. A112, 115 (1989).

22. J. Maier, Sol. State Ionics 70/71, 43 (1994).

23. K. L. Kliewer and J. S. Kohler, Phys. Rev 140, 1226 (1965).

24. K. L. Kliewer and J. S. Kohler, Phys. Rev 140, 1241 (1965).

25. K. L. Kliewer, J. Phys. Chem. Solids 27, 705 (1966).

26. A. Khandkar, V. B. Tare, and J. B. Wagner, Jr., Rev. Chim. Min. 23, 274 (1986).

27. N. Vaidehi, R Akila, A. K. Shukla, and K. T. Jacob, Mat. Res. Bull. 21, 909 (1986).

28. R. W. Ure, Jr., J. Chem. Phys. 26, 1363 (1957).

29. B. J. H. Jackson and D. A. Young, J. Phys. Chem. Solids 30, 1973 (1969).

30. C. Schlaiker and C. C. Liang, J. Electrochem. Soc. 118, 1447 (1971).

31. F. W. Poulsen, Sol. State Ionics 2, 53 (1981).

32. W. Bollman, P. Görlich, W. Hauk, and H. Mothes, Phys. Status Solidi A 2, 157 (1970);

W. Bollman and H. Henniger, ibid., A 11, 367 (1972).

33. P. W. M. Jacobs and S. H. Ong, J. Phys. 12, C7 331 (1976).

34. E. Schreck, K. Läuger, and K. Dransfield, Z. Phys. B62, 331 (1986).

35. W. G. Johnston, Phys. Rev. 98, 1777 (1955).

36. N. F. Mott, Philos. Mag. 43, 1151 (1952).

37. N. F. Mott, Philos. Mag. 44, 742 (1953).

38. A. M. Karo, J. Chem. Phys. 31, 1489 (1959).

39. W. D. Kingery, H. K. Bowen, and D. R. Ulman, Introduction to Ceramics, $2^{\text {nd }}$ Edition, (John Wiley \& Sons, New York, 1976), p. 729.

40. L. J. Schowalter and R. W. Fathauer, Crit. Rev. Sol. State Mater. Sci. 15, 367 (1989).

41. B. Wassermann, T. P. Martin and J. Maier, Sol. State Ionics 28-30, 1514 (1988).

42. B. Wassermann, W. Hönle, and T. P. Martin, Sol. State Comm. 65, 561 (1988).

\section{DISCLAIMER}

This report was prepared as an account of work sponsored by an agency of the United States Government. Neither the United States Government nor any agency thereof, nor any of their employees, makes any warranty, express or implied, or assumes any legal liability or responsibility for the accuracy, completeness, or usefulness of any information, apparatus, product, or process disclosed, or represents that its use would not infringe privately owned rights. Reference herein to any specific commercial product, process, or service by trade name, trademark, manufacturer, or otherwise does not necessarily constitute or imply its endorsement, recommendation, or favoring by the United States Government or any agency thereof. The views and opinions of authors expressed herein do not necessarily state or reflect those of the United States Government or any agency thereof. 